\title{
Cost-effectiveness of tuberculosis control strategies among immigrants and refugees
}

\author{
K. Dasgupta* and D. Menzies"
}

ABSTRACT: Today, in Western Europe, Canada and the USA, more than half of all new active tuberculosis (TB) cases occur among foreign-born migrants. This article examines the impact of migration from high TB-incidence to low TB-incidence countries, and compares the costeffectiveness of different TB control strategies.

A Medline search was conducted to identify relevant English language publications prior to December 2003. Additional articles were identified from the reference lists from these publications.

Despite the high proportion of active cases in low-incidence countries attributable to foreignborn residents, the public health impact is relatively low. Current chest radiograph screening programmes have little impact and are not cost-effective. Screening with sputum culture would improve cost-effectiveness marginally. Treatment of latent infection detected through screening with tuberculin skin testing or chest radiographs may require coercive measures to maximise impact and cost-effectiveness. In contrast, contact tracing, particularly within ethnic communities, appears to be more cost-efficient and less intrusive.

In low-incidence countries, screening of migrants at entry has little overall impact and is not a very cost-effective tuberculosis control strategy. More effective alternatives include contact tracing delivered through primary care and increased investment in global tuberculosis control.

KEYWORDS: Cost-effectiveness, migration, screening, tuberculosis control, tuberculosis diagnosis, tuberculosis prevention

$\mathrm{H}$ istorically, human migration has had a major impact on the spread of tuberculosis (TB) [1-4]. Early in the nineteenth century, 25\% of deaths in Western Europe were attributable to TB [5]. Western Europeans subsequently carried this disease to central Africa, south and Southeast Asia, and the Americas, resulting in major TB epidemics in these regions [5]. Since the 1950s, the incidence of active TB has fallen dramatically in Western European and North American countries with established market economies [1-4]. However, TB incidence remains high in most low-income countries and, in some, has even increased since the 1980s [6]. Migrants from these low-income countries continue to have relatively high rates of active TB for years after they migrate to high-income, low-incidence countries [7-9].

\section{METHODS}

A Medline search was conducted to identify English language publications prior to December 2003. The search strategy included the keyword tuberculosis, together with any of the following: migration, immigration, refugees, screening, chest radiograph, serology, seroassay, serological tests, cell-mediated immunity, tuberculin skin test, purified protein antigen, purified protein derivative, sputum culture, sputum smear, DNA amplification, PCR, cost-effectiveness, effectiveness, and
AFFILIATIONS

*Divisions of Clinical Epidemiology and Internal Medicine, Montreal General Hospital, and \#Respiratory Epidemiology and Clinical Research Unit, Montreal Chest Institute, McGill University, Montreal, QC, Canada.

CORRESPONDENCE

D. Menzies

Respiratory Epidemiology and

Clinical Research Unit

Montreal Chest Institute Montreal

QC

H2X 2 P4

Canada

Fax: 15148432083

E-mail: dick.menzies@mcgill.ca

Received:

June 182004

Accepted:

August 252004

Previous articles in this series: No. 1: Cardona P-J, Ruiz-Manzano J. On the nature of Mycobacterium tuberculosis-latent bacilli. Eur Respir J 2004; 24: 1044-1051. No. 2: Rieder H. Annual risk of infection with Mycobacterium tuberculosis. Eur Respir J 2005; 25: 181-185. No. 3: Mitchison DA. Drug resistance in tuberculosis. Eur Respir J 2005; 25: 376-379. No. 4: Kim SJ. Drug-susceptibility testing in tuberculosis: methods and reliability of results. Eur Respir J 2005; 25 : 564-569. No. 5: Dlodlo RA, Fujiwara PI, Enarson DA. Should tuberculosis treatment and control be addressed differently in HIV-infected and -uninfected individuals? Eur Respir J 2005; 25: 751-757. No. 6: Caminero JA. Management of multidrug-resistant tuberculosis and patients in retreatment. Eur Respir J 2005; 25: 928-936. 
economic analysis. Articles relevant to the topics of interest were then searched for additional references.

\section{RESULTS}

\section{Current levels of migration}

Since the 1980s, human migration has reached an unprecedented scale [10]. In 1990, the International Organization for Migration estimated that 120 million people were long-term residents of a country other than their country of birth. Just over 10 yrs later, this has increased to $>150$ million [11]. Short-term travellers are even more numerous. For example, $>50$ million foreign residents entered the USA in 2002 as short-term visitors [12] compared with 1.1 million who entered seeking permanentresident status [13].

\section{Migration and TB in low-incidence countries}

Most migrants and many visitors travel from countries where the incidence of active TB is $>40$ per 100,000 (high incidence) to countries where the incidence is $<25$ per 100,000 (low incidence) [14]. The incidence of active TB is higher among foreign-born populations than among native-born populations. In the UK, for example, the crude incidence of active TB in the native-born population in 1998 was 4.4 per 100,000 compared with $121-210$ per 100,000 in certain foreign-born populations. The increased risk among foreign-born individuals may continue for 20 yrs after migration [7-9]. As a result, in lowincidence countries, the foreign-born population account for a large proportion of reported cases of active $\mathrm{TB}$, ranging from $35-70 \%$ of all cases $[1-4,15]$.

\section{IMPACT OF TB AMONG THE FOREIGN-BORN WITHIN LOW-INCIDENCE COUNTRIES \\ Public health impact on the native-born population}

Nowadays, in most low-incidence countries, the majority of cases of active TB arise among the foreign-born; this can result in significant transmission within certain foreign-born communities in these countries [16]. However, restriction fragment length polymorphism studies have detected relatively little TB transmission from foreign-born residents to the general population $[3,17]$. The estimated proportion of active TB cases among the native-born that can be attributed to transmission from the foreign-born may be as low as $2 \%$ [18] or $11 \%$ [19], or as much as $17 \%$ [16, 20-22]. In one USA study, foreign-born TB patients were more likely to have acquired TB from USA-born individuals than vice versa [20]! A recent study indicated that $17 \%$ of active TB cases among Dutch patients were acquired from foreign-born cases, but the absolute risk in Dutch-born persons of developing active TB following transmission from a foreign-born patient was less than one in 100,000 [16]. At present, it appears that, in low-incidence countries, the overall public health impact of TB among foreign-born persons is modest.

\section{TB transmission within ethnic communities}

Many active TB cases among the foreign-born are attributable to the reactivation of latent TB infection. Reactivation rates are highest during the first 2-5 yrs following migration [1, 2, 23]. In some cases, however, active TB cases are the result of new infection acquired after migration, as demonstrated in an analysis conducted in the Netherlands of TB cases among Moroccan-origin residents [3]. In another Dutch molecular epidemiological study, isolates from all new active TB cases reported over 5 yrs demonstrated that $20-30 \%$ of all TB within several foreign-born populations was attributable to transmission within the Netherlands [16]. In the UK, even the children of foreign-born residents have higher active TB rates than the general population [24], particularly if regular visits are made to the country of origin $[24,25]$.

\section{Economic impact}

Although active TB incidence is higher among the foreign-born than within the general population, absolute rates are low, as are transmission rates. Nonetheless, the economic impact of TB among the foreign-born is substantial in low-incidence countries. In the early 1990s, total annual healthcare expenditures for TB exceeded $\$ 700$ million in the USA [26]. Given that the foreign-born now account for close to $50 \%$ of all TB cases in the USA, TB among the foreign-born accounts for more than $\$ 350$ million in healthcare expenditures annually in that country. Levels of expenditure are probably comparable in other industrialised countries, such as Canada, the UK and in Western Europe, where more than half of all cases arise among the foreign-born [2, 27].

\section{SCREENING FOR ACTIVE TB AMONG PERMANENT- RESIDENT APPLICANTS Previous and existing chest radiography screening programmes}

Screening for TB was implemented in a number of industrialised countries shortly after World War II, when refugees from Europe had high rates of active TB. These early screening programmes employed chest radiographs, which were popular in that era as a method of active detection of TB disease [15]. However, mass screening of the general population has since been abandoned, not only because the incidence in the general populations of these countries has declined, but also because it was demonstrated repeatedly that such screening had no appreciable impact on the incidence of smear-positive cases, overall morbidity or mortality $[28,29]$.

However, almost all high-income industrialised countries, with the exception of Italy, continue to utilise chest radiography screening for the detection of active TB among applicants for permanent residence [30]. In the UK, applicants undergo radiography at international ports upon arrival, and are subsequently referred to the health authority of the district of intended residence [31]. In the Netherlands, applicants undergo a screening chest radiograph within 1 week of arrival and at 6-month intervals for 2 yrs thereafter [3]. For applicants to Canada, screening chest radiographs are performed where the application is made, either overseas or within Canada [32]. Applicants with latent $\mathrm{TB}$ and an abnormal radiograph consistent with a prior TB infection (so-called "inactive $\mathrm{TB}^{\prime \prime}$ ) are referred to Canadian health authorities for follow-up after immigration [32].

\section{General considerations concerning utility of screening programmes}

Screening for a disease is justified if that disease is relatively common and treatable. The ideal screening test should be inexpensive, easy to administer, cause no discomfort to the patient, and offer both high sensitivity and specificity [33]. 
TABLE 1 Prevalence of tuberculosis (TB) among migrants to low-incidence countries

\begin{tabular}{|c|c|c|c|c|c|}
\hline \multirow[t]{2}{*}{ Authors [ref.] } & \multirow[t]{2}{*}{ Year } & \multirow[t]{2}{*}{ Population } & \multicolumn{3}{|c|}{ Prevalence \% } \\
\hline & & & Active TB & \multicolumn{2}{|c|}{ Latent TB infection } \\
\hline BLum et al. [34] & 1993 & $\begin{array}{l}\text { Amnesty programme adjustments for } \\
\text { illegal migrants from Mexico in the USA }\end{array}$ & 0.08 & 5 & 42 \\
\hline PitcheniK et al. [36] & 1982 & Haitian refugees in the USA & 0.65 & & \\
\hline Nolan et al. [37] & 1987 & Southeast-Asian refugees in the USA & 0.8 & 5.6 & 35.7 \\
\hline DASGUPTA et al. [29] & 2000 & $\begin{array}{l}\text { Permanent-resident applicants in } \\
\text { Canada }\end{array}$ & 0.15 & 2.6 & NA \\
\hline
\end{tabular}

NA: not available. \#: on the chest radiograph.

As shown in table 1 , only a small proportion of permanentresident applicants evaluated through $\mathrm{TB}$ screening programmes are found to have active pulmonary $\mathrm{TB}$ at the time of evaluation [29, 34-37]. The prevalence is higher among refugees from high-incidence countries, although still $<1 \%$ [34, $36,37]$. Conversely, the prevalence of latent infection with chest radiograph abnormalities (inactive $\mathrm{TB}$ and/or apical fibronodular disease) is substantially higher, with estimates ranging from $3-5 \%[36,37]$. Latent infection without chest radiograph abnormalities is even more common, with prevalence estimates between $35 \%$ and $42 \%$.

Effective treatments exist for both active $\mathrm{TB}$ and latent infection. While the risk of reactivation of latent TB without chest radiograph abnormalities is $\sim 0.1 \% \cdot \mathrm{yr}^{-1}[37,38]$, the reactivation risk rises to $0.2 \% \cdot \mathrm{yr}^{-1}$ in the presence of a granuloma $[39,40]$ and $0.6 \% \cdot \mathrm{yr}^{-1}$ in the presence of apical fibronodular disease [37, 41].

Two widely used screening tests for TB are the chest radiograph to detect active disease and the tuberculin skin test to detect latent infection. Sputum cultures, acid-fast staining and nucleic amplification tests are alternative tools for active TB screening. Seroassays and tests of cell-mediated immunity may have a role in the future for the identification of TB infection or disease, but these tests are still under development, and their potential utilities are unknown. The properties of these tests are reviewed below.

\section{Screening tests to detect active TB}

Chest radiograph

In the studies summarised in table 2, chest radiography had a sensitivity of $59-82 \%$ and a specificity of $52-63 \%$ for the detection of active pulmonary TB [35, 40, 42-44]. The differences in these estimates may be attributed to variations in the gold standard used (e.g. number of sputum cultures), as well as the frequency of TB and other pulmonary diseases in the populations studied. Chest radiography is less sensitive and less specific among HIV-infected individuals with advanced immunosuppression [43, 44]. If chest radiography has an overall sensitivity of $70 \%$ and specificity of $60 \%$, then,

TABLE 2 Sensitivity and specificity of chest radiography for the diagnosis of active pulmonary tuberculosis (TB)

\begin{tabular}{|c|c|c|c|c|c|}
\hline Population & $\begin{array}{l}\text { Patients hospitalised with } \\
\text { suspicion of active TB }\end{array}$ & $\begin{array}{l}\text { Patients hospitalised with } \\
\text { suspicion of active TB }\end{array}$ & $\begin{array}{l}\text { Patients hospitalised with } \\
\text { suspicion of active TB }\end{array}$ & $\begin{array}{l}\text { Individuals suspected to } \\
\text { have active TB on entry to } \\
\text { England (UK) }\end{array}$ & $\begin{array}{c}\text { Voluntary participants, } \\
\text { inner-city TB screening } \\
\text { initiative }\end{array}$ \\
\hline Gold standard & $\begin{array}{c}\text { Sputum cultures (number } \\
\text { unspecified) }\end{array}$ & $\begin{array}{c}\text { Three spontaneous sputum } \\
\text { cultures }\end{array}$ & $\begin{array}{l}\text { Up to six sputum } \\
\text { cultures, some induced }\end{array}$ & $\begin{array}{l}\text { Three sputum cultures and } \\
\text { gastric aspirate cultures }\end{array}$ & $\begin{array}{c}\text { One or two spontaneous } \\
\text { sputum cultures }\end{array}$ \\
\hline Smear positive \% & 82 & 66 & 75 & 59 & 60 \\
\hline HIV positive \% & No data & 11 & 29 & No data & No data \\
\hline $\begin{array}{l}\text { Sensitivity of } \\
\text { radiography \% }\end{array}$ & 80 & 71 & 73 & 64 & 75 \\
\hline $\begin{array}{l}\text { Specificity of } \\
\text { radiography \% }\end{array}$ & Insufficient data & 52 & 63 & 63 & 99 \\
\hline
\end{tabular}


with a prevalence of active disease of $1 \%$, chest radiography would have a positive predictive value $<1 \%$. This means that when chest radiography is used for screening for active TB in a foreign-born population, the vast majority of positive results will be falsely positive.

\section{Sputum culture}

Microbiological culture of three to six sputum samples is highly sensitive for active pulmonary TB and often serves as the gold standard for TB detection $[45,46]$. However, as shown in table 3 , the sensitivity of a single culture is comparable with that of a chest radiograph [28]. The specificity of mycobacterial culture is very high as there are very few false positives (1-2\%) resulting from cross-contamination during laboratory handling [47]. Culture results are available $\geqslant 2$ weeks after sputum sampling. Sputum may be obtained by asking the subject to cough or by inducing cough with saline aerosol, a technique termed sputum induction. In one study, culture of a single induced sputum sample was found to have greater sensitivity $(96 \%)$ than the culture of three spontaneous sputum samples (81\%) [48].

\section{Acid-fast staining}

Acid-fast staining of a sputum sample will reveal mycobacteria at a threshold of $5,000-10,000 \mathrm{bacilli} \cdot \mathrm{mL}^{-1}$ of sputum $[45,46]$. Sensitivity may be improved through changing the concentration of sputum samples and/or the use of fluorescent microscopy [45, 46]. Specificity depends on the relative prevalence of active pulmonary disease from Mycobacterium tuberculosis compared with that caused by nontuberculous mycobacteria. As indicated in table 3, acid-fast staining is more specific but less sensitive than chest radiography.

\section{Amplification tests}

These tests selectively replicate mycobacterial DNA segments. Among smear-positive cases, these tests are 95\% sensitive [4951]. However, among smear-negative, culture-positive cases, the sensitivity is lower $(50-70 \%)$ [49-52]. PCR test results are highly specific (>98\%) [49-52] and may be available within hours of sputum sampling.

\section{Immunological tests}

Most existing seroassays for TB measure immunoglobulin $G$ responses to single antigens, such as the $38-\mathrm{kDa}$ antigen. Seroassays are more sensitive for smear-positive disease than smear-negative disease, and are poorly sensitive among the immunocompromised [62]. Sensitivity (21-90\%) varies with the antigen used and the burden of disease in the population examined. No single antigen is recognised by serum antibodies from all patients $[59,63]$. Specificity is high when the control population tested includes only normal healthy volunteers, but lower when the controls have latent infection with TB. Assays that involve cocktails of antigens are presently in development [64]. Tests of cell-mediated immunity measure cytokines and interleukins or their messenger RNA precursors. One commercialised test kit is available (Quantiferon (C); Cellestis International, Carnegie, Australia), but, to date, published results are somewhat disappointing [55], although ongoing work may improve sensitivity and specificity. Results from research laboratories have demonstrated better sensitivity and specificity, which is comparable or superior to the chest

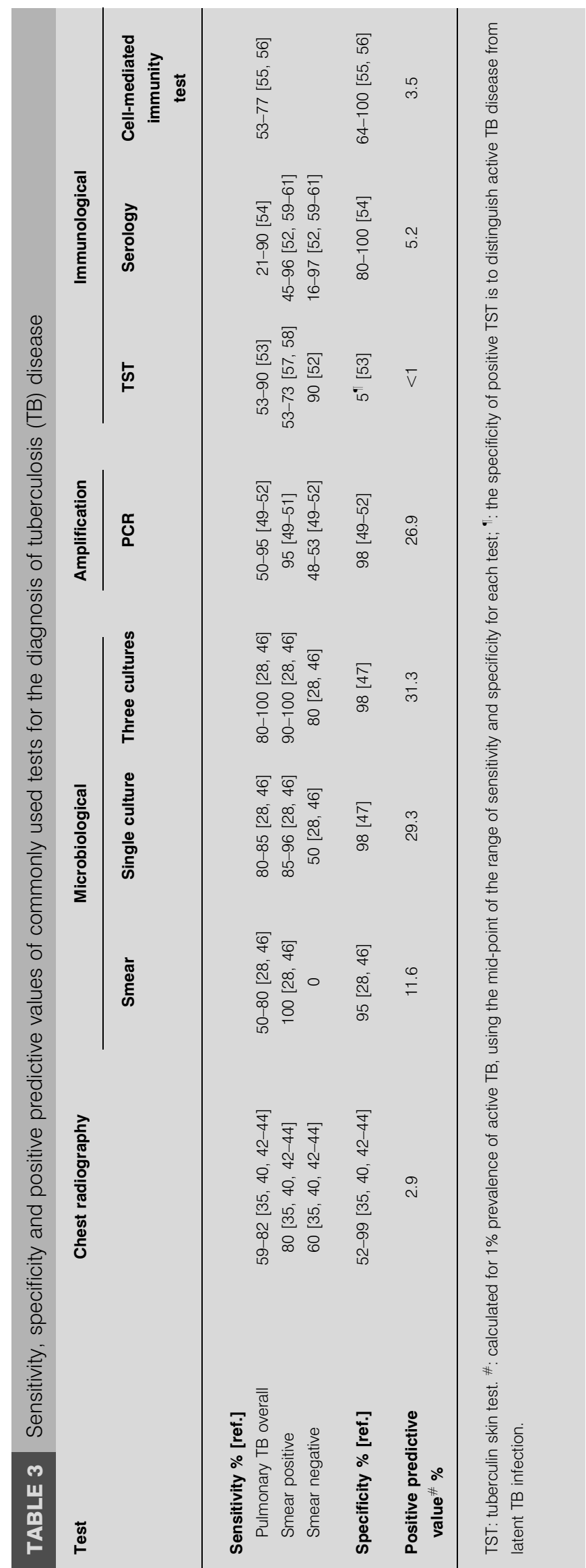


radiograph [54], but these laboratories utilise highly complex procedures with lymphocyte separation, in vitro culture and stimulation, and detection of cytokines using real-time PCR. Nevertheless, results from these laboratories suggest that these tests may be promising for the diagnosis of disease and may be able to identify, among all those with latent TB infection, those individuals who are at a particularly high risk of disease [65].

\section{Predictive values of tests for active TB}

Using average estimates of sensitivity and specificity, and a disease prevalence of $1 \%$, the positive predictive values for chest radiography, tuberculin skin test (TST), acid-fast bacilli smear, single sputum culture, multiple sputum cultures, and seroassays are shown in table 3 . The negative predictive value of all these tests is $>99 \%$. Most of these tests, including the chest radiograph, have a positive predictive value (PPV) of $<10 \%$. Sputum culture has a higher PPV, which is attributable to its greater specificity. A single sputum culture was estimated to have a PPV of $25 \%$, and three sputum cultures would result in a PPV of $33 \%$.

\section{Cost-effectiveness of a chest radiography screening programme for active TB}

Permanent-resident applicants residing in Canada at the time of application (e.g. visitors, students) undergo chest radiography screening at Canadian centres. A cost-effectiveness evaluation of chest radiography screening was conducted, using a cohort of all immigration applicants screened in the province of Quebec. During the 1-yr period examined (June 1996-June 1997), 12,898 screening chest radiographs were performed, and 722 were considered to be compatible with active TB. Seventeen applicants were confirmed to have active pulmonary $\mathrm{TB}$ through the chest radiography screening programme. Using chart review and micro-costing techniques, the cost of detection and treatment of active TB through the programme was $\$ 31,418$ (Canadian) per active case detected and treated. Conservatively estimating the cost of passive diagnosis and treatment to be $\$ 11,090$, the incremental cost of the chest radiography screening programme was $\$ 20,328$ per active case. This analysis was conducted from the perspective of the Canadian government, as Canada has a publicly funded healthcare system. Direct and indirect costs incurred by individual patients were not included [32].

\section{Cost-effectiveness of alternative tools for active TB screening}

The costs of alternative screening tests, as outlined in table 4, were derived from published sources and estimates provided by the laboratories of the McGill University Health Centre (Montréal, Canada). The material costs of nucleic acid amplification assays are six-fold higher than the other tests that were considered. Labour costs for both amplification tests and sputum culture are considerable, particularly if sputum is induced. Using these cost figures and cohort data from a previous study [32], rough estimates of the cost to detect each active case from screening using different tests are shown in table 5. Although the cost of the screening test itself would be higher using sputum culture, the cost per active case of TB disease detected would be lower with a single sputum culture than with chest radiography. Similarly, despite the low cost for

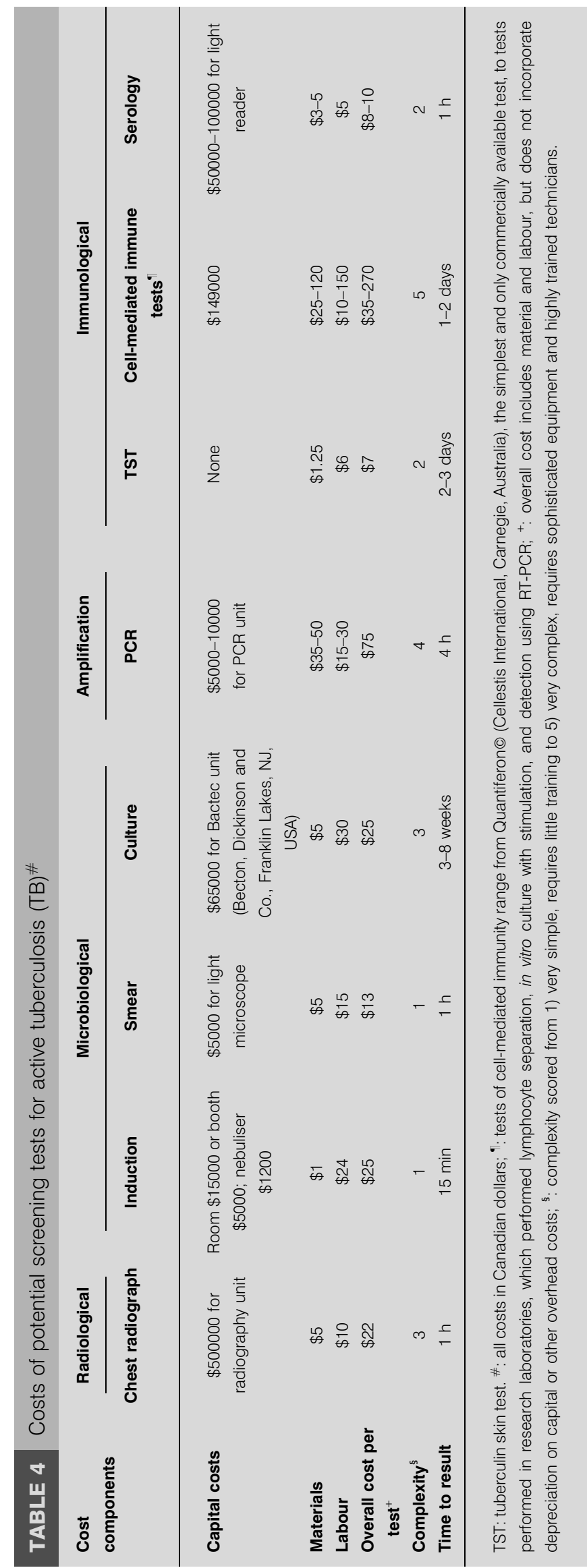




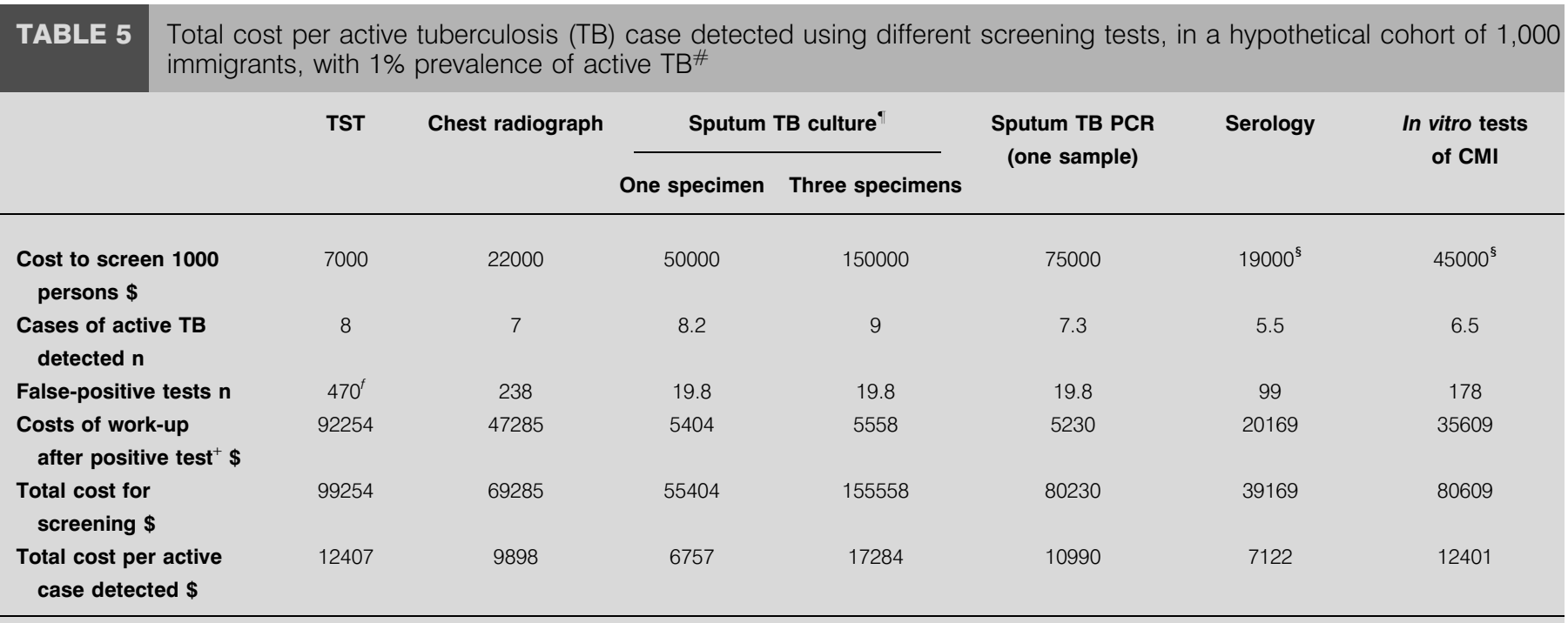

TST: tuberculin skin test; CMI: cell-mediated immunity. \#: all costs in Canadian dollars; ": sputum TB culture includes cost of sputum induction, but does not include acid-fast bacilli smear; ${ }^{+}$: average costs were $\$ 193$ for the evaluation of persons with positive screening test in a chest specialist clinic [32], and costs do not include overhead, administration or patient costs; ${ }^{\text {s: }}$ : serology and CMI include the cost of drawing blood samples $(\$ 10)$; ${ }^{f}$ : assume that the prevalence of positive TST would be $50 \%$.

initial screening with TSTs or serology, overall costs to detect each active case would be higher because of their poor specificity. This is because screening with a more specific test, such as sputum culture, would result in a much smaller number of false-positive tests. This would result in much lower costs for the further evaluation of individuals with falsepositive tests. This is important because the second step of medical evaluation and further investigation is more expensive than all screening tests.

\section{SCREENING FOR LATENT TB INFECTION}

The TST involves the intradermal injection of purified protein antigen, with measurement of the resulting induration within 48-72 h. Development of induration requires a cell-mediated immune response. The TST is the diagnostic test used to define latent infection and is considered to be the most sensitive test for this condition. However, when the TST is used in patients with active TB, $10-47 \%$ will have a falsely negative TST [52, 57, 58], and the size of the TST reaction is not useful for distinguishing between prevalent active disease and latent infection [66].

The use of the TST as the primary screening test in the USA is consistent with a shift in emphasis from the detection of prevalent active TB to the detection of latent infection. The US Institute of Medicine has recommended TST testing among all immigrants from countries with high TB incidence [67]. The prevalence of latent infection among permanent-resident applicants is 30-75\% [67]. Future risk of TB may be reduced through preventive treatment with agents such as isoniazid $[42,68]$. The specificity of the TST for latent infection can range 60-90\% [53] with false-positive tests due to previous bacillus Calmette-Guérin vaccination or exposure to environmental, nontuberculous mycobacteria, both of which are common among applicants from developing countries [69].
A recent cost-effectiveness analysis concluded that tuberculin screening of all immigrants and refugees to the USA would result in net cost savings to the American society. In this analysis, a hypothetical cohort of immigrants entering the USA during 2000 was constructed. The analytical horizon was the expected lifetime of the cohort. Using a decision analysis model, the investigators calculated that, without TST screening, 13,933 active TB cases would occur, but, with screening and isoniazid treatment, 4,342 cases would occur. The net cost without TST screening would be $\$ 338$ million, while the cost with TST screening would be $\$ 258$ million. There would, therefore, be a net saving of $\$ 8,320$ per case prevented. Patientincurred costs (transportation, time) were included in this analysis, increasing costs associated with active disease because of the loss of time attributable to hospitalisation. However, administrative costs and the costs that would be incurred to institute a TB screening programme were not included in the analysis. Such costs would substantially increase the cost of a TST screening programme. In addition, the completion rates for latent TB infection (LTBI) did not take into account the following: refusal to undergo tuberculin testing; failure of those with positive TST to report for medical evaluation; physician noncompliance with guidelines for the prescription of therapy; and refusal of patients to start the therapy. In several reports from large-scale screening programmes, these problems resulted in a very substantial reduction in overall programme effectiveness, as only $11-30 \%$ of individuals with a positive TST completed an adequate course of LTBI therapy [34, 70-74].

The TST should be more sensitive in detecting latent infection, because chest radiographs will be abnormal in only $10-20 \%$ of those with latent infection. However, the subgroup of individuals with latent TB infection who have abnormal chest radiographs are at an increased risk of reactivation. Therefore, 
chest radiography screening, followed by TST screening, may be more cost-effective if this results in the treatment of fewer individuals with latent infection but who have a much higher risk of reactivation.

This approach has been examined in a study of the cost per active TB case prevented through chest radiography screening in Canada. Among 722 permanent-resident applicants with chest radiograph abnormalities identified over a 1-yr period, 353 were diagnosed as having latent infection: that is, TST was positive, chest radiograph abnormalities were stable, and sputum cultures were negative. A Markov model was used to examine the number of active TB cases prevented through treatment of these patients. In this model, the yearly probability of reactivation among TST reactors varied with chest radiograph abnormalities (no TB-compatible changes $0.1 \%$, granuloma $0.2 \%$, fibronodular disease $0.6 \%$ ). Among these 353 patients, 145 actually completed preventive therapy, resulting in the prevention of 7.85 cases of active TB. The incremental cost per active TB case prevented was $\$ 39,409$. All programme costs, including administrative costs, were included in this calculation. Had $90 \%$ of eligible patients been prescribed preventive treatment and $80 \%$ been compliant, the incremental cost per case prevented would have decreased to $\$ 21,240$. The analysis was conducted from the perspective of the government. Patient-incurred costs were not included [32].

An alternative scenario compared the chest radiograph versus TST as the primary screening tool among immigrants to Canada, a low-incidence country. This study relied on Markov modelling and was conducted from the perspective of the Canadian government. The costs borne by patients were not included. In this analysis, the annual risk of TST reactors was set at $0.1 \%$ for those without chest radiograph abnormalities and $0.66 \%$ for those with TB-compatible abnormalities. If the prevalence of latent TB infection was $50 \%$, chest radiography screening was projected to prevent 1.2 active cases per 1,000 individuals screened, whereas TST screening would prevent 2.9 cases per 1,000 individuals screened. The incremental cost per case prevented was $\$ 10,627$ for chest radiography screening compared with $\$ 66,750$ for TST screening. Higher costs of the TST screening strategy were attributed to the identification of a larger number of low-risk, as well as false-positive, reactors as candidates for chemoprophylaxis.

\section{ALTERNATIVE APPROACHES TO SCREENING AT ENTRY}

The evaluation of close contacts of active pulmonary TB cases has been established as a cost-effective method for detecting as well as preventing active TB. Studies in the UK have estimated that $1 \%$ of all contacts had active disease at the time of initial screening, and that $10 \%$ of all active TB cases were identified through contact screening [75-77]. A contact-tracing programme in Quebec resulted in an incremental \$815 saving per active case detected, because of the relatively high number of active cases detected (six cases among 103 infected contacts of 244 identified) and the reduced need for hospitalisation compared with a passive detection strategy [32]. Contact tracing also resulted in incremental savings of $\$ 600$ per active case prevented, because of the detection of a large number of individuals with LTBI and the high rate of completion of LTBI therapy among these individuals [32].
A recent study conducted in an economically deprived area of the UK with a large proportion of Bangladeshi immigrants demonstrated that contact tracing was more effective than new-entrant screening. Among 263 contacts of smear-positive index cases, 13 cases of active TB were diagnosed compared with no cases detected among 322 new entrants screened. Interestingly, when 227 contacts of nonpulmonary index active TB cases were evaluated, two active TB cases were identified. The investigators suggested that, although nonpulmonary TB is not transmissible, it may identify communities or families at an increased risk (e.g. high-density housing, frequent return trips to country of origin) [78].

\section{LIMITATIONS OF SCREENING OF IMMIGRANTS AND REFUGEES}

One of the greatest limitations of screening new immigrants and refugees is that screening is performed only once at the time of initial entry, and only for individuals who seek permanent-resident status. However, permanent residents make frequent return visits, and there are far more foreignborn migrants entering industrialised countries under other legal statuses. Of all foreign-born entrants to the USA, only $2 \%$ seek permanent residence. The remainders are students, migrant labourers, individuals visiting friends and family in the USA, other categories of visitor, and persons who enter illegally. All of these may stay for many years, but they are not screened at entry. Permanent residents may also return to their country of origin, often doing so repeatedly. In two studies conducted in England, it was estimated that $20-30 \%$ of all TB cases among foreign-born permanent residents were due to reexposure during these return visits [24, 25]. Such cases will not be prevented by screening programmes.

\section{CONCLUSIONS}

The magnitude of human migration from high TB-incidence regions to low-incidence regions has reached an unprecedented scale. Foreign-born individuals currently account for the majority of active TB cases in most low-incidence countries. The costs of treating these individuals are substantial. While rates of transmission to the general population are low, transmission within specific ethnic communities of lowincidence countries may be higher.

When considering the most cost-effective approach for the control of TB in foreign-born migrants, it is important to remember that cost-effectiveness is affected by the perspective of the analysis (government, private payer, patient, society), costs of services (evaluations, tests, hospitalisation, transportation), and the effectiveness of available interventions. Existing TB screening programmes for migrants to low TB-incidence countries have used chest radiography to detect active TB in permanent-resident applicants. Due to the very low prevalence of active TB in this population and the low PPV of the chest radiograph, radiographic screening for active $\mathrm{TB}$ at entry has minimal impact and is not cost-effective. The major potential benefit of screening at entry is the detection of individuals with LTBI and abnormal chest radiographs, but only if they receive preventive therapy. This means that the screening programme must have the capacity to provide treatment for LTBI. Given the current recommended standard of isoniazid treatment for 9 months, a substantial infrastructure is necessary to ensure 
adequate compliance, and to ensure that adverse events, which can rarely be fatal, are detected and managed promptly. This increases the expense and complexity of any screening programme. Nevertheless, the detection and treatment of inactive TB through chest radiography will be more costeffective than the detection of latent infection through TST, but neither is highly cost-efficient.

The replacement of chest radiography screening with sputum culture would offer a small improvement in cost-effectiveness, but would not detect latent infection. Tests of cell-mediated immunity and seroassays involving cocktails of antigens are emerging technologies that offer the potential to both detect and differentiate active and LTBI. However, these new technologies have not been evaluated for screening purposes and, at the present time, are generally more costly than chest radiograph or TST, so their utility and cost-effectiveness remain unclear at this time.

Effective TB screening strategies are also needed for all other entrants, as well as for permanent residents who return home to their high-incidence countries. Designing screening programmes that address all of these potential sources of TB infection is likely to be complex and expensive. A more effective use of resources may be comprehensive contact tracing within foreign-born communities through local primary care networks.

The ideal long-term tuberculosis control strategy would be global investment to improve tuberculosis control in highincidence countries. If successful, this could result in a global reduction in tuberculosis incidence, which would reduce the risk of tuberculosis among human migrants travelling from high tuberculosis-incidence to low tuberculosis-incidence regions. Such a strategy would be more humanitarian and may be more cost-effective than the current approaches to tuberculosis control among these migrants.

\section{REFERENCES}

1 Rieder H, Zellweger J, Raviglione M, Keizer ST, Migliori GB. Tuberculosis control in Europe and international migration. Eur Respir J 1994; 7: 1545-1553.

2 Euro TB (inVS/KNCV) and National coordinators for tuberculosis surveillance in the WHO European Region, Surveillance of tuberculosis in Europe - Euro TB. Report on tuberculosis cases notified in 2001. Saint-Maurice, Institut de veille sanitaire, 2003; pp. 1-95.

3 Bwire R, Nagelkerke N, Keizer ST, et al. Tuberculosis screening among immigrants in The Netherlands: what is its contribution to public health? Neth J Med 2000; 56: 63-71.

4 Long R, Njoo H, Hershfield E. Tuberculosis: 3. Epidemiology of the disease in Canada. CMAJ 1999; 160: 11851190.

5 Davies PD. Tuberculosis and migration. The Mitchell Lecture 1994. J R Coll Physicians Lond 1995; 29: 113-118.

6 Dye C, Scheele S, Dolin P, Pathania V, Raviglione MC. Global burden of tuberculosis: estimated incidence, prevalence and mortality by country. JAMA 1999; 282: 677-686.
7 Gaudette L, Ellis E. Tuberculosis in Canada: a focal disease requiring distinct control strategies for different risk groups. Tuber Lung Dis 1994; 24: 244-253.

8 Zuber PL, McKenna MT, Binkin NJ, Onorato IM, Castro KG. Long-term risk of tuberculosis among foreign-born persons in the United States. JAMA 1997; 278: 304-307.

9 National survey of notifications of tuberculosis in England and Wales in 1983. Medical Research Council Tuberculosis and Chest Diseases Unit. BMJ (Clin Res Ed) 1985; 291: 658-661.

10 Menzies D. Tuberculosis crosses borders. Int J Tuberc Lung Dis 2000; 4: Suppl. 2, S153-S159.

11 International Organization for Migration. www.iom.int/ iomwebsite. Date last accessed: August 152004.

12 International Trade Administration: Office of Travel and Tourism Industries. http://tinet.ita.doc.gov/. Date last accessed: April 15 2004. Date last updated: March 152005.

13 U.S. Citizenship and Immigration Service. http://uscis. gov/graphics/shared/aboutus/statistics/index.htm. Date last accessed: April 15 2004. Date last updated: March 18 2005.

14 Joint Tuberculosis Committee of the British Thoracic Society. Control and prevention of tuberculosis in the United Kingdom: code of practice 2000. Thorax 2000; 55: 887-901.

15 Binkin NJ, Zuber PL, Wells CD, Tipple MA, Castro KG. Overseas screening for tuberculosis in immigrants and refugees to the United States: current status. Clin Infect Dis 1996; 23: 1226-1232.

16 Borgdorff MW, Nagelkerke $\mathrm{N}$, van Soolingen $\mathrm{D}$, de Haas PEW, Veen J, van Embden JDA. Analysis of tuberculosis transmission between nationalities in the Netherlands in the period 1993-1995 using DNA fingerprinting. Am J Epidemiol 1998; 147: 187-195.

17 Dahle UR, Sandven P, Heldal E, Caugant DA. Continued low rates of transmission of Mycobacterium tuberculosis in Norway. J Clin Microbiol 2003; 41: 2968-2973.

18 Chin DP, DeRiemer K, Small PM, et al. Differences in contributing factors to tuberculosis incidence in U.S.-born and foreign-born persons. Am J Respir Crit Care Med 1998; 158: 1797-1803.

19 Musana K, Menzies D, Tannenbaum TN, et al. Molecular epidemiology of tuberculosis in 3 Canadian cities, 1995-97. Am J Respir Crit Care Med 1999; 159: A904.

20 Jasmer RM, Ponce de Leon A, Hopewell PC, et al. Tuberculosis in Mexican-born persons in San Francisco: reactivation, acquired infection and transmission. Int $J$ Tuberc Lung Dis 1997; 1: 536-541.

21 Small PM, Hopewell PC, Singh SP, et al. The epidemiology of tuberculosis in San Francisco. A population-based study using conventional and molecular methods. N Engl J Med 1994; 330: 1703-1709.

22 Tornieporth NG, Ptachewich Y, Poltoratskaia N, et al. Tuberculosis among foreign-born persons in New York City, 1992-1994: implications for tuberculosis control. Int J Tuberc Lung Dis 1997; 1: 528-535.

23 ten Asbroek AH, Borgdorff MW, Nagelkerke NJ, et al. Estimation of serial interval and incubation period of tuberculosis using DNA fingerprinting. Int J Tuberc Lung Dis 1999; 3: 414-420. 
24 Ormerod LP, Green RM, Gray S. Are there still effects on Indian Subcontinent ethnic tuberculosis of return visits?: a longitudinal study 1978-97. J Infect 2001; 43: 132-134.

25 McCarthy OR. Asian immigrant tuberculosis: the effect of visiting Asia. Br J Dis Chest 1984; 78: 248-253.

26 Brown RE, Miller B, Taylor WR, et al. Health-care expenditures for tuberculosis in the United States. Arch Intern Med 1995; 155: 1595-1600.

27 Health Canada. Tuberculosis Statistics 2002. No. H49-108/ 2002. Ontario, Public Health Agency of Canada, 2003.

28 Toman K. Tuberculosis - Case-finding and chemotherapy: questions and answers. Geneva, World Health Organization, 1979.

29 Horwitz O, Darrow M. Principles and effects of mass screening: Danish experience in tuberculosis screening. Public Health Rep 1976; 91: 146-153.

30 Rapiti E, Fano V, Forastiere F, et al. Determinants of tuberculosis in an immigrant population in Rome: a casecontrol study. Int J Tuberc Lung Dis 1998; 2: 479-483.

31 Van den Bosch CA, Roberts JA. Tuberculosis screening of new entrants; how can it be made more effective? J Public Health Med 2000; 22: 220-223.

32 Dasgupta K, Schwartzman K, Marchand R, Tannenbaum TN, Brassard P, Menzies D. Comparison of cost effectiveness of tuberculosis screening of close contacts and foreign-born populations. Am J Respir Crit Care Med 2000; 162: 2079-2086.

33 Sackett DL, Haynes RB, Tugwell P. Clinical Epidemiology. Toronto, Little, Brown, 1985.

34 Blum RN, Polish LB, Tapy JM, Catlin BJ, Cohn DL. Results of screening for tuberculosis in foreign-born persons applying for adjustment of immigration status. Chest 1993; 103: 1670-1674.

35 Markey AC, Forster SM, Mitchell R, Larson E, Smith H. Suspected cases of pulmonary tuberculosis referred from port of entry into Great Britain, 1980-3. BMJ 1986; 292: 378-379.

36 Pitchenik AE, Russell BW, Cleary T, Pejovic I, Cole C, Snider D Jr. The prevalence of tuberculosis and drug resistance among Haitians. N Engl J Med 1982; 307: 162-165.

37 Nolan CM, Elarth AM. Tuberculosis in a cohort of Southeast Asian refugees. Am Rev Respir Dis 1988; 137: 805-809.

38 Comstock GW, Edwards LB, Livesay VT. Tuberculosis morbidity in the US Navy: its distribution and decline. Am Rev Respir Dis 1974; 110: 572-580.

39 Horwitz O, Wilbek E, Erickson PA. Epidemiological basis of tuberculosis eradication. 10. Longitudinal studies on the risk of tuberculosis in the general population of a lowprevalence area. Bull World Health Organ 1969; 41: 95-113.

40 Grzybowksi S, McKinnon NE, Tuters L, Pinkus G, Philipps R. Reactivations in inactive pulmonary tuberculosis. Am Rev Respir Dis 1966; 93: 352-360.

41 Grzybowski S, Fishaut H, Rowe J, Brown A. Tuberculosis among patients with various radiologic abnormalities, followed by the chest clinic service. Am Rev Respir Dis 1971; 104: 605-608.

42 Barnes PF, Verdegem TD, Vachon LA, Leedom JM, Overturf GD. Chest roentgenogram in pulmonary tuberculosis: new data on an old test. Chest 1988; 94: 316-320.
43 Tattevin P, Casalino E, Fleury L, Egmann G, Ruel M, Bouvet E. The validity of medical history, classic symptoms, and chest radiographs in predicting pulmonary tuberculosis: derivation of a pulmonary tuberculosis prediction model. Chest 1999; 115: 1248-1253.

44 Cohen R, Muzaffar S, Capellan J, Azar H, Chinikamwala M. The validity of classic symptoms and chest radiographic configuration in predicting pulmonary tuberculosis. Chest 1996; 109: 420-423.

45 Long R. Canadian tuberculosis standards. Toronto, Canadian Lung Association, 2000.

46 American Thoracic Society. Diagnostic standards and classification of tuberculosis in adults and children. Am Rev Respir Dis 2000; 161: 1376-1395.

47 Burman WJ, Stone BL, Reves RR, et al. The incidence of false-positive cultures for Mycobacterium tuberculosis. Am J Respir Crit Care Med 1997; 155: 321-326.

48 Makkar H, Mallampalli A, Matthew M, et al. Improved yield of Mycobacterium tuberculosis with sputum induction in addition to three expectorated sputa. Am J Respir Crit Care Med 2000; 161: A298.

49 Abe C, Hirano K, Wada M, et al. Detection of Mycobacterium tuberculosis in clinical specimens by polymerase chain reaction and the gen-probe amplified mycobacterium tuberculosis direct test. J Clin Microbiol 1993; 31: 3270-3274.

50 Clarridge JE, Shawar RM, Shinnick TM, Plikaytis BB. Large-scale use of polymerase chain reaction for detection of Mycobacterium tuberculosis in a routine mycobacteriology laboratory. J Clin Microbiol 1993; 31: 2049-2056.

51 Jonas V, Alden MJ, Curry JI, et al. Detection and identification of Mycobacterium tuberculosis directly from sputum sediments by amplification rRNA. J Clin Microbiol 1993; 31: 2410-2416.

52 Al-Zahrani K, Jahdali HA, Poirier L, Rene P, Gennaro ML, Menzies D. Accuracy and utility of commercially available amplification and serologic tests for the diagnosis of minimal pulmonary tuberculosis. Am J Respir Crit Care Med 2000; 162: 1323-1329.

53 Menzies D. The tuberculin skin test. In: Reichman L, Hershield E, eds. Tuberculosis, a comprehensive international approach. New York, Dekker M, 2000.

54 Daniel T, Oxtoby M, Pinto E, Moreno E. The immune spectrum in patients with pulmonary tuberculosis. Am Rev Respir Dis 1981; 123: 556-559.

55 Mazurek G, LoBue PA, Daley CL, Bernardo J, Lardizabal AA, Bishai WR. Comparison of a whole-blood interferon gamma assay with tuberculin skin testing for detecting latent Mycobacterium tuberculosis infection. JAMA 2001; 256: 1740-1747.

56 Anderson P, Munk ME, Pollock S, Doherty TM. Specific immune-based diagnosis of tuberculosis. Lancet 2000; 356 1099-1104.

57 Holden M, Dubin MR, Diamond PH. Frequency of negative intermediate-strength tuberculin sensitivity in patients with active tuberculosis. N Engl J Med 1971; 285: 1506-1509.

58 Harrison BDW, Tugwell P, Fawcett IW. Tuberculin reaction in adult Nigerians with sputum-positive pulmonary tuberculosis. Lancet 1975; 1: 421-424. 
59 Bothamley GH, Rudd R, Festenstein F, Ivanyi J. Clinical value of the measurement of Mycobacterium tuberculosis specific antibody in pulmonary tuberculosis. Thorax 1992; 47: 270-275.

60 Harboe M, Wiker HG. The 38-kDa protein of Mycobacterium tuberculosis: a review. J Infect Dis 1992; 166: 874-884.

61 Jackett PS, Bothamley GH, Batra HV, Mistry A, Young DB. Specificity of antibodies to immunodominant mycobacterial antigens in pulmonary tuberculosis. J Clin Microbiol 1988; 26: 2313-2318.

62 Chan ED, Heifets L, Iseman MD. Immunologic diagnosis of tuberculosis: a review. Tuber Lung Dis 2000; 80: 131-140.

63 Lyashchenko K, Colangeli R, Houde M, Al Jahdali H, Menzies D, Gennaro ML. Heterogeneous antibody responses in tuberculosis. Infect Immun 1998; 66: 3936-3940.

64 Gennaro ML. Immunologic diagnosis of tuberculosis. Clin Infect Dis 2000; 30: Suppl. 3, S243-S246.

65 Doherty TM, Demissie A, Olobo J, et al. Immune responses to the Mycobacterium tuberculosis-specific antigen ESAT-6 signal subclinical infection among contacts of tuberculosis patients. J Clin Microbiol 2002; 40: 704-706.

66 Al-Zahrani K, Al Jahdali H, Menzies D. Does size matter? Utility of size of tuberculin reactions for the diagnosis of mycobacterial disease. Am J Respir Crit Care Med 2000; 162: 1419-1422.

67 Institute of Medicine (US). Ending neglect: the elimination of tuberculosis in the United States. Washington, National Academy Press, 2000.

68 American Thoracic Society. Targeted tuberculin testing and treatment of latent tuberculosis infection. This official statement of the American Thoracic Society was adopted by the ATS Board of Directors, July 1999. This is a Joint Statement of the American Thoracic Society (ATS) and the Centers for Disease Control and Prevention (CDC). This statement was endorsed by the Council of the Infectious Diseases Society of America. (IDSA), September 1999, and the sections of this statement. Am J Respir Crit Care Med 2000; 161: S221-S247.

69 Schwartzman K, Menzies D. Tuberculosis screening of immigrants to low-prevalence countries. Am J Respir Crit Care Med 2000; 161: 780-789.

70 Adhikari N, Menzies R. Community-based tuberculin screening in Montreal: a cost-outcome description. Am J Public Health 1995; 85: 786-790.

71 Yuan L, Richardson E, Kendall PRW. Evaluation of a tuberculosis screening program for high-risk students in Toronto schools. CMAJ 1995; 153: 925-932.

72 Jereb J, Etkind SC, Joglar OT, Moore M, Taylor Z. Tuberculosis contact investigations: outcomes in selected areas of the United States, 1999. Int J Tuberc Lung Dis 2003; 7: Suppl. 3, S384-S390.

73 Saraiya M, Cookson ST, Tribble P, et al. Tuberculosis screening among foreign-born persons applying for permanent US residence. Am J Public Health 2002; 92: 826-829.

74 BC Center for Disease Control. Annual Report Tuberculosis Control in 2002. Vancouver, BC Ministry of Health, 2003.

75 Ormerod LP. Results of tuberculosis contact tracing: Blackburn 1982-90. Respir Med 1993; 87: 127-131.

76 Esmonde TF, Petheram IS. Audit of tuberculosis contact tracing procedures in south Gwent. Respir Med 1991; 85: 421-424.

77 Kumar S, Innes JA, Skinner C. Yield from tuberculosis contact tracing in Birmingham. Thorax 1992; 47: 875.

78 Underwood BR, White VL, Baker T, Law M, MooreGillon JC. Contact tracing and population screening for tuberculosis: who should be assessed? J Public Health Med 2003; 25: 59-61. 